\title{
CERULOPLASMIN PLASMA LEVELS IN PATIENTS WITH SEVERE FORMS OF HERPES INFECTION
}

\author{
Irina Novikova, Maria Zlotnikova*
}

\author{
Department of Clinical Laboratory Diagnostics, Gomel State Medical University, Lange st., 5, Gomel, Belarus \\ E-mail: ustinovamv@bk.ru
}

Received: May 16, 2011; Accepted with revision: September 22, 2011; Available online: November 1, 2011

Key words: Herpes infection/Ceruloplasmin/C-reactive protein

Background. Research interest in ceruloplasmin (CP) has significantly increased in recent years owing to new discoveries of its properties including anti-inflammatory and anti-oxidant effects. Data on CP blood plasma level in patients with herpetic infection caused by herpes simplex virus are scarce and often contradictory. Most point to a reduction of $\mathrm{CP}$ in the blood plasma of patients during the exacerbation of the disease with gradually return to normal values after treatment. There is evidence of an increase in CP levels during the acute period of CRHI (chronic recurrent herpes infection) and decrease in remission.

Materials and methods. The content of ceruloplasmin in blood plasma was determined by immunoturbidimetry using the test-systems "Spinreact" (Spain) and biochemical analyzer "Architect C8000".

Results. We found that in patients with severe forms of chronic recurrent herpes infection in the exacerbation period, $\mathrm{CP}$ levels were increased by approximately $35 \%$ relative to the control values. However in the remission period in $80 \%$ of patients, CP concentrations remained elevated and in some patients the CP level was even increased in comparison with values in the exacerbation. Such dynamics of CP were not caused by the exacerbation of concomitant diseases.

Conclusion. The increased CP levels in the remission period in patients with severe forms of chronic recurrent herpes infection may have been caused by its effect as an endogenous antioxidant. No correlations of CP levels with other laboratory signs of acute inflammation were found but identified was a relation to free radical activity.

\section{INTRODUCTION}

Ceruloplasmin (CP), the copper carrying glycoprotein related to the $\alpha 2$ globulin fraction of blood serum, transports ions of copper from the liver to various organs and tissues for the synthesis of copper-containing enzymes. Recently the interest of researchers in this protein has increased owing to new discoveries. Ceruloplasmin is a representative of the group of acute phase inflammation proteins (so called "weak reactant"). Its synthesis in the liver increases by $20-60 \%$ under the influence of proinflammatory cytokines (especially IL-6) (ref. ${ }^{1-3}$ ). As an acute phase protein, $\mathrm{CP}$ acts as a mediator of the immune system participating in the reactions of antigen elimination, localization of lesions and development of reparative processes. On the cells of several organs and tissues, including immune cells, receptors to $\mathrm{CP}$ are found through which it acts ${ }^{4-7}$. Ceruloplasmin is capable of potentiating the microbicidal activity of phagocyte cells in the inflammatory response through the release of free iron ions at lower $\mathrm{pH}\left(\right.$ ref. $\left.^{8}\right)$.

On the other hand, ceruloplasmin may have an antiinflammatory effects connected to its antioxidant properties $^{3,8,9}$. Due to its ferroxidase activity, CP oxidizes $\mathrm{Fe}^{2+}$ to $\mathrm{Fe}^{3+}$ preventing the participation of $\mathrm{Fe}^{2+}$ in the Haber-Weiss reaction and the subsequent initiation of lipid peroxidation. Further CP is able to neutralize the superoxide anion radical providing superoxide dismutase- like action, and acts, in fact, as an extracellular superoxide dismutase $\mathrm{e}^{8,10-13}$.

In view of the information above, $\mathrm{CP}$ is now considered as one of the most important components of innate immunity.

The data on CP levels in the blood plasma of patients with herpetic infections caused by herpes simplex virus is scarce and often contradictory. Most studies point to the reduction of CP in the blood plasma of patients during the exacerbation of the disease with gradual return to normal values after treatment. There is also evidence on the increase in CP levels during the acute period of CRHI (chronic recurrent herpes infection) and decrease in the remission period $^{14,15}$.

The purpose of this study was to analyse blood plasma levels of ceruloplasmin in patients with chronic recurrent herpes infection.

\section{MATERIALS AND METHODS}

\section{Patients}

We examined 108 patients (21 men and 87 women, mean age $32 \pm 11$ years) with severe forms of chronic recurrent herpes infections (CRHI) treated in the "Republican research center of radiation medicine and human ecology" (Gomel, Belarus). The criteria for severe forms assumed to be more than 6 recurrences per year 
and the spreading character of rashes. The duration of the disease ranged from 1 to 24 years. In 40 patients, exacerbation of the disease was diagnosed, 68 patients were in the remission period.

In all the examined patients, concomitant chronic infections and inflammatory diseases were found. These included 72 persons with respiratory tract diseases (chronic tonsillitis, chronic rhinitis, chronic pharyngitis, chronic bronchitis simplex), and 49 persons with urogenital tract diseases (chronic bacterial interstitial nephritis, urolithiasis, prostatitis, cystitis, vaginitis). However, at the time of the examination, no exacerbation of concomitant diseases was noted. Patients with concomitant diseases of the cardiovascular system, diabetes mellitus, and diseases of the liver were excluded from the investigation. The control group consisted of 40 healthy persons ( 5 males and 35 females) of comparable age.

\section{Ceruloplasmin concentration}

We used peripheral blood, taken from the cubital vein into a test tube with heparin $(10 \mathrm{U} / \mathrm{ml})$. The concentration of ceruloplasmin in blood plasma was determined by the immunoturbidimetric method using the test-systems "Spinreact" (Spain) and biochemical analyzer "Architect C8000”.

\section{Statistics}

Statistical analysis of the results was performed using the software package "Statistica 6.0". Taking into account the tests for normality of distribution we used the non parametric methods: Mann-Whitney U-test (for unrelated groups), Wilcoxon W-test (for related groups), Spearman test (for correlation analysis), contingency tables $2 \times 2\left(\chi^{2}\right)$ for the frequency analysis. The differences were considered statistically significant at $\mathrm{p}<0.05$. Data were presented as medians and interquartile interval $(25 ; 75 \%)$.

\section{RESULTS}

\section{The concentration of ceruloplasmin in blood plasma} of patients with CRHI

The concentration of $\mathrm{CP}$ in blood plasma, according to different authors, has a wide range of normal values. Hence, in the first stage of the study we established the reference interval. For this we formed a control group under the acceptance of stringent criteria of clinical laboratory diagnosis to exclude factors that could affect the value of this parameter. The control group consisted of volunteers from the "Republican research center of radiation medicine and human ecology" (Gomel), as well as students of 5-6 courses of Gomel State Medical University. Volunteers filled in the questionnare which included a list of diseases, associated, as a rule, with the change of plasma CP level (viral and non viral hepatitis, cholestasis, cirrhosis, arterial hypertension, atherosclerosis of coronary and peripheral arteries, ischemic heart disease, diabetes mellitus, toxic goiter, malignant neoplasm, rheumatoid arthritis, anemia, and others). Patients with at least one of the listed diseases, were excluded from the examination, the remaining volunteers underwent general and biochemical blood analysis. Patients with no abnormalities in laboratory parameters formed the control group. In the formed control group, the concentration of CP ranged between 259-345 mg/1 (25-75\%), 210-405 mg/1 (5-95\%), 193-411 mg/1 (Min-Max). For CP levels in patients with herpes infection, the interquartile interval scale was (25; $75 \%)$. The range of $\mathrm{CP}$ concentration in healthy patients was accepted as the norm.

We compared the levels of CP in blood plasma of patients with $\mathrm{CRHI}$, examined during the exacerbation $(n=40)$ and during the remission $(n=68)$.

It should be noted that the compared groups did not differ significantly by sex or age or clinical manifestations of disease (Table 1 ).

The plasma levels of ceruloplasmin in patients who were examined both in the exacerbation and in the remission was significantly higher than the levels of the control group (increasing degree $35 \%$ and $42 \%$ respectively;

Table 1. Clinical characteristics of patients with severe forms of CRHI.

\begin{tabular}{|l|c|c|}
\hline \multicolumn{1}{|c|}{ Parameter } & $\begin{array}{c}\text { Patients with CRHI examined } \\
\text { during the exacerbation, } \mathrm{n}=40\end{array}$ & $\begin{array}{c}\text { Patients with CRHI examined } \\
\text { during the remission, } \mathrm{n}=68\end{array}$ \\
\hline Age, years & $35(26 ; 46)$ & $32(23 ; 43)$ \\
\hline Men, women, \% & $22 / 78$ & $18 / 82$ \\
\hline Duration of CHRI, years & $4(2 ; 7)$ & $6(4 ; 8)$ \\
\hline Exacerbations per year (\% of patients): & & 61 \\
- from 6 to 11 exacerbations per year & 57 & 39 \\
- more than 12 exacerbations per year & 43 & 68 \\
\hline Localization of the lesions (\% of patients): & & 32 \\
\hline - nazolabial herpes & 65 & \\
- combined lesions (labial and anogenital) & 35 & \\
\hline
\end{tabular}




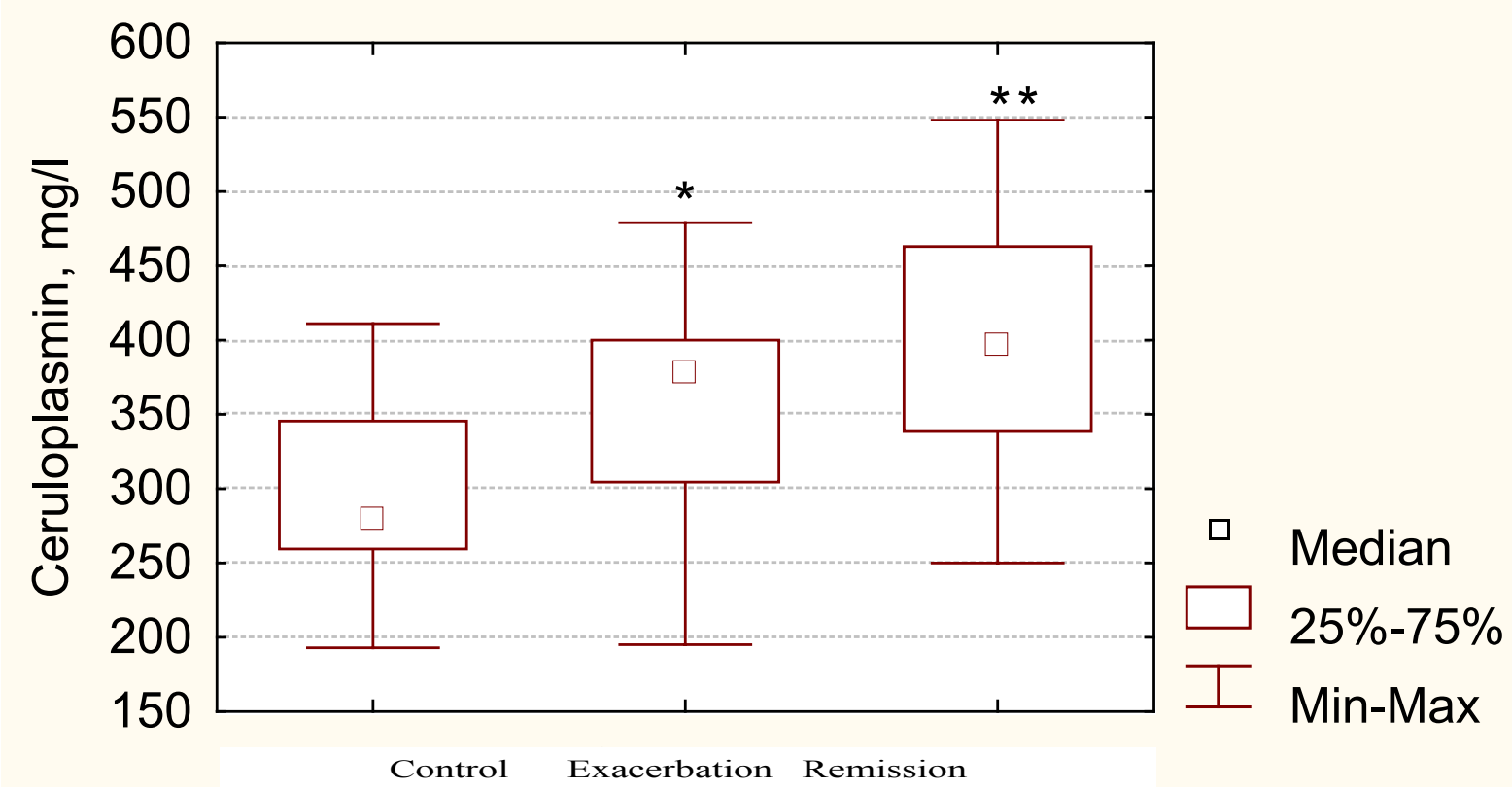

Fig. 1. Concentration of $\mathrm{CP}$ in the blood plasma of patients with $\mathrm{CRHI}$ in the exacerbation and the remission periods of the disease.

*The differences were statistically significant compared with the control group $(\mathrm{p}<0.05)$.

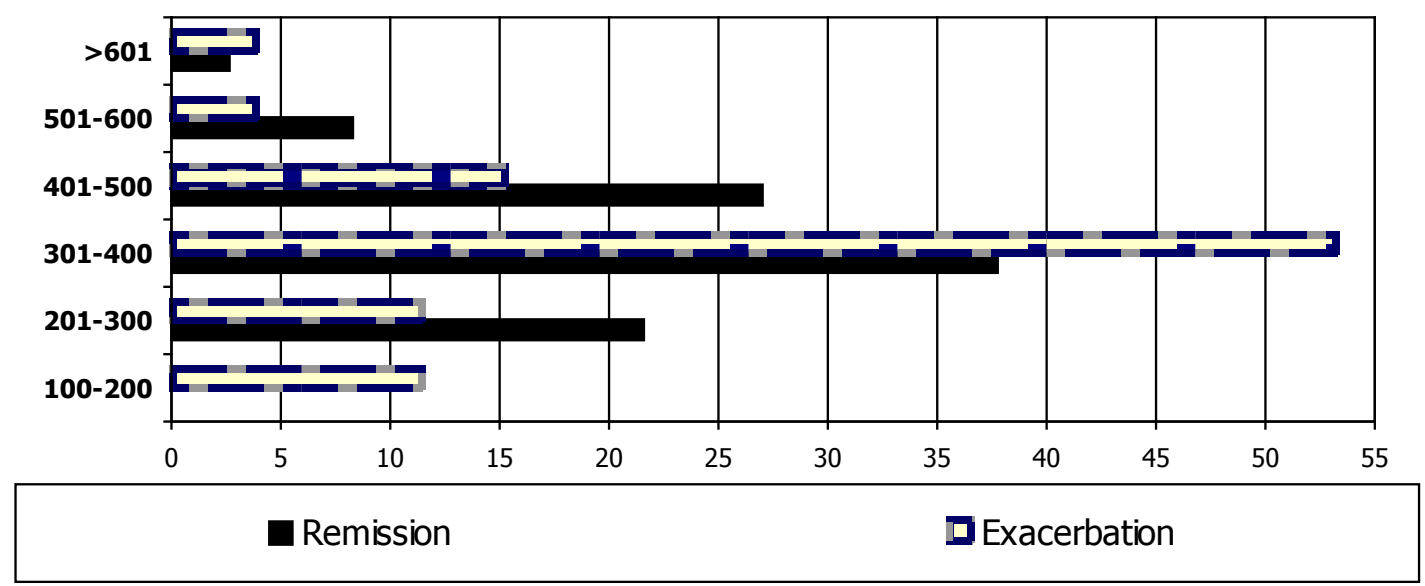

Fig. 2. The profile of $\mathrm{CP}$ levels in patients with CRHI.

In axis $\mathrm{X}$ - number of patients (\%); in axis $\mathrm{Y}$ - the range of $\mathrm{CP}$ concentration in $\mathrm{mg} / \mathrm{l}$.

$\mathrm{p}=0.001$ and $\mathrm{p}<0.001)$ (Fig. 1). The level of $\mathrm{CP}$ in patients in the remission period was the highest (the differences between the compared groups were significant, $p=0.04$ ).

As the examined patients had a wide range of $\mathrm{CP}$ levels in the plasma, we analyzed the frequency of cases with different CP levels (Fig. 2). The analysis was performed taking into account the range of $100 \mathrm{mg} / \mathrm{l}$. This was a step change we thought appropriate since in the control group the interquartile interval $(25 ; 75 \%)$ of $\mathrm{CP}$ concentration did not exceed $100 \mathrm{mg} / \mathrm{l}$.

As shown in (Fig. 2), in the majority of patients examined in the exacerbation of the disease, the concentrations of CP were in the range of $301-400 \mathrm{mg} / 1$ (53\% of examined persons). In the range of $200-500 \mathrm{mg} / 1$ there were $87 \%$ patients in remission and $79 \%$ in exacerbation.
In patients examined in the exacerbation period, high concentrations of $\mathrm{CP}$ (above the physiological values) were revealed more rarely $(4 \%)$, than decreased concentrations $(12 \%)\left(\chi^{2}=18 ; p<0.001\right)$.

It is interesting to note that the persons examined in the remission period of CRHI had no CP concentrations below $200 \mathrm{mg} / 1$, whereas among patients examined in the exacerbation period of the disease this was revealed in $12 \%$.

In 34 patients, we analyzed the changes in the dynamics of the infectious process (exacerbation $\rightarrow$ remission). All patients had a severe course of infection with a frequency of exacerbations - 6 to 14 times per year, and a duration of disease - from 3 to 19 years. The dynamic monitoring was carried out as follows: the first exami- 
Table 2. Some laboratory tests of patients in the dynamics of the infectious process.

\begin{tabular}{|l|c|c|}
\hline \multicolumn{1}{|c|}{ Test } & $\begin{array}{c}\text { Patients with CRHI examined } \\
\text { during the exacerbation, } \mathrm{n}=34\end{array}$ & $\begin{array}{c}\text { Patients with CRHI examined } \\
\text { during the remission, } \mathrm{n}=34\end{array}$ \\
\hline Leukocytes, $10^{9} / 1$ & $5.80(4.84 ; 7.50)$ & $5.32(4.65 ; 6.72)$ \\
\hline Lymphocytes, $10^{9} / 1$ & $1.96(1.63 ; 2.40)$ & $1.8(1.45 ; 2.36)$ \\
\hline Erythrocytes, $10^{12} / 1$ & $4.51(4.30 ; 4.83)$ & $4.31(4.23 ; 4.80)$ \\
\hline ESR, $\mathrm{mm} / \mathrm{h}$ & $10(6 ; 12)$ & $7(4 ; 11)$ \\
\hline Total protein, $\mathrm{g} / 1$ & $74(65 ; 77)$ & $69(64 ; 75)$ \\
\hline C-reactive protein, $\mathrm{mg} / 1$ & $3.2(1.1 ; 4.8)$ & $2.6(0.5 ; 4.3)$ \\
\hline Ceruloplasmin, $\mathrm{mg} / 1$ & $403(344 ; 451)$ & $435(389 ; 447)$ \\
\hline Transferrin, $\mathrm{g} / 1$ & $2.49(1.83 ; 2.72)$ & $2.27(1.76 ; 2.61)$ \\
\hline Ferritin, $\mathrm{ng} / \mathrm{ml}$ & $45.8(14.8 ; 124.0)$ & $57.2(17.8 ; 113.6)$ \\
\hline
\end{tabular}

*Differences between groups are absent.

nation at the time of CRHI exacerbation on the stage of formed vesicles (prior to the appointment of antiviral drugs) and reexamination - in the remission period ( 2 weeks after completion of the combined therapy).It should be noted that in the examined patients, despite the presence of herpetic lesions there were no laboratory signs of inflammation. Comparison analysis of some laboratory tests of patients in the dynamics of the infectious process is shown in (Table 2).

It was found that $\mathrm{CP}$ levels in the exacerbation period in these patients in the whole group were increased relative to the levels of healthy patients (increasing degree: $43 \%$; $p=0.02$ ), and under achieving clinical remission remained high as well (increasing degree relative to healthy individuals was $54 \%$; $p=0.03$ ).

The individual analysis of the CP dynamics revealed that in 16 patients, the level of $\mathrm{CP}$ during the transition of the process from the exacerbation into the remission period increased by more than $100 \mathrm{mg} / 1$, in 11 patients it remained virtually unchanged (changes in $10-70 \mathrm{mg} / \mathrm{l}$ ), and in 7 patients there was a significant decrease in the concentration of $\mathrm{CP}$. The differences in the frequency of increasing and decreasing $\mathrm{CP}$ levels were statistically significant. $\left(\chi^{2}=5.3 ; p=0.021\right)$. No differences in clinical parameters between these patients (frequency of recurrence, localization of lesions, concomitant diseases) were found.

Next, we analyzed changes in CP levels in the dynamics of the natural course of herpes infections. We examined 11 patients twice with an interval of 3 months under the scheme "remission $\rightarrow$ remission". During this time, the patients received antiviral drugs if it was necessary (in case of exacerbation) but within 5-6 days prior to the examination, the drugs were not taken. It was found that in 5 of 11 patients the variations of $\mathrm{CP}$ levels in the reexamination did not exceed $100 \mathrm{mg} / 1(20-70 \mathrm{mg} / \mathrm{l})$, whereas in 6 patients there were significantly more pronounced variations $(110-440 \mathrm{mg} / 1)$. The latter had more low frequencies of exacerbations than patients with low dynamics of $\mathrm{CP}$ $(9(6 ; 11)$ and $16(10 ; 22)$ respectively; $\mathrm{p}=0.039)$.
In the next stage of the study we analyzed the concentrations of CP in relation to clinical features of the disease (disease duration, frequency of exacerbations, localization of lesions, presence of concomitant diseases and burdened allergic history, intestinal dysbiosis). In this case, for the patients examined in the remission of the disease, a correlation between the plasma levels of CP and the frequency of exacerbations was found. In patients with an almost continuous course of herpetic infection (more than 12 exacerbations per year) in comparison with patients with the lower frequency of exacerbations, CP levels were significantly higher $(489 \mathrm{mg} / 1$ and $358 \mathrm{mg} / 1$ respectively, $\mathrm{p}=0.04$ ). When analyzing the other above mentioned clinical features in patients examined in the remission, significant differences in levels of $\mathrm{CP}$ were not found. The correlation coefficient also confirmed the association of CP level with frequency of exacerbation of HSV infections in patients examined in the remission period of the disease $(r=0.36 ; p=0.04)$.

In patients examined in the exacerbation of CRHI we found a correlation between the plasma level of CP and the duration of the disease history. When the duration of the disease was more than 5 years, CP level was significantly lower than for patients with a shorter term of the disease $(314 \mathrm{mg} / 1$ and $372 \mathrm{mg} / 1$ respectively, $\mathrm{p}=0.04$ ). When analyzing the other clinical features in patients who were examined in the exacerbation period, no significant differences in CP levels were found. Correlation analysis also confirmed an association of CP level with the duration of disease history in patients examined in the remission period of the disease $(\mathrm{r}=-0.41 ; \mathrm{p}=0.05)$.

In previous studies we demonstrated that in patients with CRHI both in the exacerbation and in the remission periods, activation of lipid peroxidation and increase in the spontaneous production of oxygen radicals by neutrophils were observed, that is, the intensification of free radical oxidation ${ }^{16}$. Taking into account the antioxidant properties of $\mathrm{CP}$, we analyzed the correlations between $\mathrm{CP}$ level and the parameters of free radical oxidation us- 
ing a Spearman correlation coefficient. It was found that in patients who were in the remission period of CRHI, CP levels positively correlated with the concentration of primary peroxidation products (dien conjugates) of erythrocytes phospholipids $(\mathrm{r}=0.61 ; \mathrm{p}=0.04)$ and secondary oxidation products (trien conjugates) of neutral lipids $(\mathrm{r}=0.54 ; \mathrm{p}=0.02)$, as well as the spontaneous ability of blood neutrophils to develop reactive oxygen species by the nitroblue tetrazolium test $(r=0.40 ; p=0.03)$. In healthy individuals and patients with exacerbation of CRHI these relationships were absent. The correlations of CP concentration with laboratory signs of inflammation like ESR, leukocyte amount, C-reactive protein, both in the acute stage and in remission of the disease, were not found.

\section{DISCUSSION}

In our study we found that in patients with chronic recurrent herpes infection of a severe course the plasma level of CP in most cases (79-87\%) was within physiological norms ( 180 to $500 \mathrm{mg} / 1$ ), but was increased relatively to the interquartile interval of the control group.

As is well known, well-formed reference interval is the key to the reliability of laboratory information. In our study the control group was composed of healthy individuals of age and sex similar to the examined patients with CRHI. In addition, through means of questionnaires and laboratory tests (complete blood count, routine biochemical analysis), we excluded volunteers with clinical laboratory signs of immunological reactivity disorders and with diseases, which could significantly affect the change of $\mathrm{CP}$ concentrations (liver diseases, severe cardiovascular system diseases, diabetes mellitus and others). In this case the interquartile interval of CP levels in healthy individuals was rather narrow (259-345 mg/1), which allowed us to perform an adequate analysis of $\mathrm{CP}$ changes in patients with CRHI. We revealed that the level of CP was increased both during the exacerbation and the remission.

As is known, ceruloplasmin is an acute phase protein (the so called "weak reactant"), and its concentration increases by $20-60 \%$ at the beginning of the acute inflammation process. Therefore the increase of CP levels in plasma of patients with CRHI, examined during the exacerbation period of the disease is logical. However the absence of correlations between CP level increase and the concentration of other indicators of acute process (ESR, leukocytes amount, the concentration of C-reactive protein) cast doubt on the hypothesis about increasing of $\mathrm{CP}$ level in the exacerbation period of CRHI as an acute phase protein.

It remains unclear why there was no reduction in $\mathrm{CP}$ levels during the transition to the stage of clinical remission. It is known that $\mathrm{CP}$ has a short half-life (about 6 days), so it is reasonable to assume its gradual decrease during the attenuation of the inflammatory process. At the same time, examination of the dynamics of herpetic infection course (according to the scheme "exacerbationremission" and "remission-remission") did not reveal such changes, and the character of $\mathrm{CP}$ level changes did not depend on the stage of the process. This may be the result of a constant antigenic load caused by viral persistence or delayed transformation of CP after the acute phase of inflammation. However, we showed that in some patients, the concentration of $\mathrm{CP}$ in the remission period even increased compared with the period of exacerbation. This necessitates the search for other causes of pronounced fluctuations of CP concentration, as indicated by other authors $^{9,17,18}$.

One of the reasons for increased CP levels in patients with CRHI in the remission period may be its participation in the process of free radical oxidation. It is known that CP has pronounced antioxidant activity and it is capable of inactivating superoxide radicals and reducing the possibility of $\mathrm{OH}$-generating reactions ${ }^{16}$. Taking into account the previously demonstrated activation of lipid peroxidation in patients with CRHI, who remain in remission, as well as the activation of superoxide anion production by neutrophils in these patients, such mechanism for $\mathrm{CP}$ increasing seems quite reasonable ${ }^{16}$. It is additionally confirmed by the presence of moderate correlations between CP concentrations and levels of primary products of erythrocyte phospholipid peroxidation and secondary products of plasma neutral lipids oxidation, as well as with the spontaneous ability of blood neutrophils to develop reactive oxygen species by nitroblue tetrazolium test.

Thus, these data indicate that the most probable reason for increased CP levels in patients with severe CRHI in between exacerbation periods is its antioxidant activity. The clinical importance of CP concentration monitoring in patients with recurrent herpetic infection has yet to be established.

\section{CONCLUSION}

We have shown that in patients with severe forms of chronic recurrent herpes infection in the exacerbation period, CP levels were increased by approximately $35 \%$ relative to the control values. However in the remission period in $80 \%$ of patients CP concentrations remained elevated and in some patients the CP level was even higher than the values in the exacerbation. Such dynamics of $\mathrm{CP}$ were not caused by the exacerbation of concomitant diseases. Correlations of CP levels with other laboratory signs of acute inflammation (ESR, leukocytosis, level of C-reactive protein) were not detected but the correlation with the activity of the free radical process (parameters of lipid peroxidation, production of oxygen radicals by neutrophils in blood) was found. This allows us to assume that increased CP levels in the remission period may been caused by its effect as an endogenous antioxidant.

\section{REFERENCES}

1. Mohammad G, Mishra VK, Pandey HP. Antioxidant of some nanoparticles may enhance wound healing in T2DM patients. Digest J Nanomaterials and Biostructures 2008;4:159-62.

2. Musci G. Structure/function relationships in ceruloplasmin. Adv Exp Med Biol 1999;448:175-82. 
3. Halliwell B, Gutteridge JM. The antioxidants of human extracellural fluids. Arch Biochem Biophys 1990;1:1-8.

4. Biron CA, Brossay L. NK cells and NKT cells in innate defense against viral infections. Curr Opin Immunol 2001;13:458-64.

5. Bellner L, Thoren F, Nygren E. A proinflammatory peptide from herpes simplex virus type 2 glycoprotein $\mathrm{G}$ affects neutrophil, monocyte, and NK cell functions. J Immunol 2005;174(4):2235-41.

6. Gordon S. Alternative activation of macrophages. Nat Rev Immunol 2003;3:23-35.

7. Lutsenko S, Gupta A, Burkhead JL, Zuzel V. Cellular multitasking: the dual role of human $\mathrm{Cu}$-ATPases in cofactor delivery and intracellular copper balance. Arch Biochem Biophys 2008;476:22-32.

8. Atanasiu RL, Stea D, Mateescu MA. Direct evidence of ceruloplasmin antioxidant properties. Mol cell Biochem 1998;189:127-35.

9. Guhrer Orhan H, Ozgunes H, Beksac MS. Correlation between plasma malondialdehyde and ceruloplasmin activity values in preeclamptic pregnancies. Clin Biochem 2000;34:505-6.

10. Gutteridge JMC, Halliwell B.H. Free radicals and antioxidants in the year 2000. A historical look to the future. Ann Ny Acad SCI 2000;899:136-47.

11. Gutteridge JMC, Quinlan GJ. Antioxidant protection against organic and inorganic oxygen radicals by normal human plasma: The important primary role for iron-binding and iron-oxidising proteins. Biochem Biophys Ada 1992;1159:248-54.
12. Kim IG, Park SY. Requirement of intact human ceruloplasmin for the glutathione-linked peroxidase activity. FEBS Letters 1998;437:293-6.

13. Volchegorsky IA, Nalimov AG, Yarovinsky BG. Different mesns of lipid peroxidation products estimation in heptane-isopropanole extract of blood. Vopr Med Chem (Moscow) 1989;1:127-30 (in Russian).

14. Suvorov AP, Voronina TN. Correction antioxidative system on herpetic infection. Rus Jornal Skin and Venerol Dis (Moscow) 2003;4:15-6 (in Russian).

15. Nagoev BS, Kambachokova ZA. The state of the processes of lipid peroxidation and the antioxidant system in patients with recurrent herpetic infection. Inf Dis (Moscow) 2010;8:2.27-9 (in Russian).

16. Zlotnikova MV, Novikova IA. Functional neutrophils activity and lipid peroxidation in patients with severe form of herpes infection. Probl of Health and Ecology (Belarus) 2011;1:57-63 (in Russian).

17. Virit O, Selek S, Bulut M, Savas HA, Celik H, Erel O. High ceruloplasmin levels are associated with obsessive compulsive disorder: a case control study. Behav Brain Funct 2008;18:47-52.

18. Giurgea N, Constantinescu MI, Stanciu R, Suciu S, Muresan A. Ceruloplasmin - acute-phase reactant or endogenous antioxidant? The case of cardiovascular disease. Med Sci Monit 2005;11(2):48-51. 